Open Communications in Nonlinear Mathematical Physics

]ocnmp

Vol.1 (2021) pp 57 66] ARTICLE

\title{
Reciprocal Transformations in Relativistic Gasdynamics. Lie Group Connections
}

\author{
Sergey V. Meleshko ${ }^{1}$ and Colin Rogers ${ }^{2}$ \\ ${ }^{1}$ School of Mathematics, Institute of Science, Suranaree University of Technology, \\ Thailand \\ ${ }^{2}$ School of Mathematics and Statistics, The University of New South Wales, Sydney, \\ NSW2052, Australia
}

Received April 14, 2021; Accepted July 13, 2021

\begin{abstract}
Reciprocal transformations associated with admitted conservation laws were originally used to derive invariance properties in non-relativistic gasdynamics and applied to obtain reduction to tractable canonical forms. They have subsequently been shown to have diverse physical applications to nonlinear systems, notably in the analytic treatment of Stefan-type moving boundary problem and in linking inverse scattering systems and integrable hierarchies in soliton theory. Here,invariance under classes of reciprocal transformations in relativistic gasdynamics is shown to be linked to a Lie group procedure.
\end{abstract}

\section{Introduction}

Bateman [1] in a study of lift and drag aspects in two-dimensional homentropic irrotational gasdynamics established invariance of the governing system under a novel multi-parameter class of relations which have come to be known as reciprocal transformations. The latter, characteristically are associated with conservation laws admitted by a system. The reciprocal transformations of [1] were subsequently discussed by Tsien [2] in connection with the application of the Kármán-Tsien model pressure-density law which, in turn, has its roots in the classical work of Chaplygin [3] on gas jets. Thus, with a Kármán-Tsien law, the class of reciprocal transformations derived by Bateman may be applied to link, in subsonic régimes, a gasdynamic system with an associated hydrodynamic system encapsulated in a Cauchy-Riemann system. Loewner [4] later in this gasdynamics context sought via infinitesimal Bäcklund transformations to construct systematically model gas laws which asymptotically lead to such a canonical reduction. It is remarkable that contained therein, mutatis mutandis is a linear representation to be re-discovered some twenty years later for

(C) The author(s). Distributed under a Creative Commons Attribution 4.0 International License 
the sine Gordon equation. It accordingly constitutes a seminal connection between modern soliton theory and the subsequent applications therein of Bäcklund transformations [5, 6].

It was established in [7] that the kind of infinitesimal transformations introduced by Loewner in [4 may likewise be applied in Lagrangian nonlinear elastodynamics. In that case, novel model stress-strain laws were derived which are linked to an integrable sinhGordon equation and which, importantly, admit an interior change of concavity. This material phenomenon can occur, for instance, in the compression of polycrystalline materials [8] and in the unloading régimes of superelastic nickel-titanium [9, 10].

In [11, 12, a re-interpretation and extension of the infinitesimal Bäcklund transformations of [4] was shown to lead to a linear representation for a wide class of 2+1-dimensional solitonic equations. The $\bar{\partial}$-dressing method was outlined for these generalised Loewner systems. In particular, a novel canonical 2+1-dimensional sine Gordon equation was derived which, like the Davey-Stewartson and Nizhnik-Novikov-Veselov equations contain the two spatial variables with equal standing.

In soliton theory, reciprocal transformations associated with admitted conservation laws and their incorporation in Bianchi diagrams linked to auto-Bäcklund transformations were introduced in [13. Reciprocal transformations in 1+1-dimensions have been applied in the linkage of the AKNS and WKI inverse scattering schemes [14] and in a range of solitonic contexts (see e.g. [15]-[20] and literature cited therein). In [21] reciprocal transformations in 2+1-dimensions were applied to connect the Kadomtsev-Petviashvili, modified Kadomtsev-Petviashvili and Dym integrable hierarchies. The links therein were formulated as Darboux theorems for associated Lax operators.

In nonlinear continuum mechanics, reciprocal transformations have likewise proved to have diverse physical applications. In [22, 23], they have been applied to solve moving boundary problems which arise in the modelling of melting in a range of metals as described in the seminal work of Storm 24. Nonlinear moving boundary problems arising in sedimentation theory [25], the percolation of liquids through porous media such as soils [26], and methacrylate saturation processes [27] have likewise been solved via the application of reciprocal transformations. In recent work, reciprocal transformations have been applied in 28] in connection with moving boundary problems associated with the solitonic Dym equation. It is recalled that a link has been established in [29, 30] between a classical Saffman-Taylor problem with interface moving at constant speed and certain solutions of the canonical Dym equation 31.

The preceding attests to the importance of reciprocal transformations in physical applications. In [32], a novel link was established between a one-parameter subclass of infinitesimal reciprocal-type transformations in gasdynamics and a Lie group approach. Thereby, a standard one-parameter $(\beta)$ class of reciprocal transformations may be reconstructed via a standard Lie group procedure involving solution of a system of initial value problems. The identity transformation is retrieved in the limit $\beta \rightarrow \infty$. In [33], this one-parameter class of reciprocal transformations was applied to a 1+1-dimensional Lagrangian anisentropic system with a Prim-type gas law associated with the affinsphären equation which arises in the theory of Tzitzeica surfaces [34, 35. An integrable deformed version of this solitonic affinsphären equation was obtained thereby and a linear representation set down.

In [36, 37, invariance under multi-parameter reciprocal transformations has recently been established in relativistic gasdynamics. Here, a connection between one-parameter 
subclasses of such transformations and a Lie group procedure is established.

\section{Reciprocal invariance in 1+1-dimensional relativistic gas- dynamics}

In [38], a reciprocal invariance property was established as embodied in the following:

Theorem 2.1. The relativistic 1+1-dimensional gasdynamic system

$$
\begin{aligned}
\frac{\partial}{\partial t}\left(\frac{\rho c}{\sqrt{c^{2}-v^{2}}}\right)+\frac{\partial}{\partial x}\left(\frac{\rho c v}{\sqrt{c^{2}-v^{2}}}\right) & =0, \\
\frac{\partial}{\partial t}\left(\frac{(e+p) v}{c^{2}-v^{2}}\right)+\frac{\partial}{\partial x}\left(\frac{(e+p) v^{2}}{c^{2}-v^{2}}+p\right) & =0
\end{aligned}
$$

is invariant under the 4-parameter class of reciprocal transformations

$$
\begin{gathered}
\rho^{*}=\frac{a_{3} \rho \sqrt{\left(p+a_{2}\right)^{2}-a_{1}^{2} v^{2} / c^{2}}}{\sqrt{1-v^{2} / c^{2}}}\left[\frac{1}{p+\left((e+p) / c^{2}\right) v^{2} /\left(1-v^{2} / c^{2}\right)+a_{2}}\right], \\
v^{*}=-\frac{a_{1} v}{p+a_{2}}, \\
e^{*}=\frac{a_{3} S\left(p+a_{2}\right)\left(1-a_{1}^{2} v^{2} / c^{2}\left(p+a_{2}\right)^{2}\right)}{p+S v^{2}+a_{2}} c^{2}-a_{4}+\frac{a_{1}^{2} a_{3}}{p+a_{2}} \\
p^{*}=a_{4}-\frac{a_{1}^{2} a_{3}}{p+a_{2}}
\end{gathered}
$$

with

$$
a_{1} d t^{*}=S v d x-\left(p+S v^{2}+a_{2}\right) d t, \quad d x^{*}=d x
$$

and $S=(e+p) /\left(c^{2}-v^{2}\right)$.

The above result in the limit $c \rightarrow \infty$ reproduces the 4-parameter class of reciprocal invariant transformations originally obtained in 1+1-dimensional non-relativistic gasdynamics in [38]. Cognate adjoint-type invariant transformations have application in the analysis of gas flow between a piston and a non-uniform shock in [39].

In [36], a one-parameter sub-class of reciprocal transformations which constitute a specialisation of those in [38] was reconstructed via application of Lie group methods involving an associated Cauchy initial value problem. Here, the subclass of reciprocal transformations (21)-(3) for the system (11) is considered with

$$
\begin{gathered}
a_{1}=-\epsilon^{-1}, \quad a_{2}=a_{4}=\epsilon^{-1} \\
a_{3}=1
\end{gathered}
$$


and accordingly becomes

$$
\begin{gathered}
\rho^{*}=\frac{\rho \sqrt{(\epsilon p+1)^{2}-v^{2} / c^{2}}}{\left(\epsilon\left(p+\left((e+p) / c^{2}\right) v^{2} /\left(1-v^{2} / c^{2}\right)\right)+1\right) \sqrt{1-v^{2} / c^{2}}}, \\
v^{*}=\frac{v}{\epsilon p+1}, p^{*}=\frac{p}{\epsilon p+1} \\
e^{*}=\frac{S\left(c^{2}(\epsilon p+1)^{2}-v^{2}\right)}{\left(\epsilon\left(p+S v^{2}\right)+1\right)(\epsilon p+1)} c^{2}-\frac{p}{\epsilon p+1}, \\
d t^{*}=-\epsilon\left(S v d x-\left(p+S v^{2}\right) d t\right)+d t, \quad d x^{*}=d x
\end{gathered}
$$

It is readily verified that the one-parameter class of relations (5)-(6) constitute a Lie group. Moreover, the reciprocal relations (5)-(6) satisfy the Cauchy problem

$$
\begin{aligned}
\frac{d \rho^{*}}{d \epsilon} & =-\rho^{*} v^{* 2} e^{*} \Gamma^{* 2}, & & \rho_{\mid \epsilon=0}^{*}=\rho, \\
\frac{d v^{*}}{d \epsilon} & =-p^{*} v^{*}, & & v_{\mid \epsilon=0}^{*}=v, \\
\frac{d p^{*}}{d \epsilon} & =-p^{* 2}, & & p_{\mid \epsilon=0}^{*}=p, \\
\frac{d e^{*}}{d \epsilon} & =\left(c^{2} p^{* 2}-v^{* 2} e^{* 2}\right) \Gamma^{* 2}, & & e_{\mid \epsilon=0}^{*}=e, \\
\frac{d\left(d t^{*}\right)}{d \epsilon} & =S^{*} v^{*} d x^{*}-\left(p^{*}+S^{*} v^{* 2}\right) d t^{*}, & & \left(d t^{*}\right)_{\mid \epsilon=0}=d t, \\
\frac{d\left(d x^{*}\right)}{d \epsilon} & =0, & & \left(d x^{*}\right)_{\mid \epsilon=0}=d x,
\end{aligned}
$$

where $\Gamma^{*}=\left(c^{2}-v^{* 2}\right)^{1 / 2}$. Similar to the classical theory of a Lie group of point transformations, it is convenient to present them by the infinitesimal generator

$$
X=-\rho v^{2} e \Gamma^{2} \partial_{\rho}-p v \partial_{v}-p^{2} \partial_{p}+\left(c^{2} p^{2}-e^{2} v^{2}\right) \Gamma^{2} \partial_{e}+\left(\left(c^{2} p+e v^{2}\right) d t-v(e+p) d x\right) \Gamma^{2} \partial_{d t} .
$$

An advantageous aspect of this infinitesimal approach is that it reveals hidden invariants under the reciprocal transformations. In this connection, a function $J$ is an invariant of the group of transformations (5), (6) if and only if it satisfies the differential equation [40]

$$
X J=0 .
$$

A particular triad of such invariants is given by

$$
\begin{gathered}
J_{1}=\frac{(c p-v e)(c-v)}{(c p+v e)(c+v)}, \quad J_{2}=\frac{\rho p}{(c p+e v)} \sqrt{\frac{c-v}{c+v}}, \\
J_{3}=\frac{v(c p+e v)}{p(c-v)\left(p c^{2}+e v^{2}\right)}\left(\left(c^{2} p+e v^{2}\right) d t-v(e+p) d x\right) .
\end{gathered}
$$

It is remarked that the above procedure to construct one-parameter invariant transformations may, in principle, be extended by application of the infinitesimal generator

$$
Y=\zeta^{\rho} \partial_{\rho}+\zeta^{v} \partial_{v}+\zeta^{p} \partial_{p}+\zeta^{e} \partial_{e}+\left(\left(c^{2} p+e v^{2}\right) d t-v(e+p) d x\right) \Gamma^{2} \partial_{d t},
$$


wherein the functions $\zeta^{\rho}, \zeta^{v}, \zeta^{p}$ and $\zeta^{e}$ depend on $(\rho, v, p, e)$. This is under present investigation.

\section{Reciprocal invariance in two-dimensional relativistic gas dynamics}

In [37] the following invariance result was established:

Theorem 3.1. The two-dimensional relativistic gasdynamic system

$$
\begin{aligned}
\partial_{x}(R u)+\partial_{y}(R v) & =0, \\
\partial_{x}\left(p+S u^{2}\right)+\partial_{y}(S u v) & =0, \\
\partial_{x}(S u v)+\partial_{y}\left(p+S v^{2}\right) & =0, \\
\partial_{x}(S u)+\partial_{y}(S v) & =0,
\end{aligned}
$$

where

$$
q^{2}=u^{2}+v^{2}, \quad \Gamma=\frac{1}{\sqrt{c^{2}-q^{2}}}, \quad R=\rho \Gamma, \quad S=\frac{e+p}{c^{2}} \Gamma^{2},
$$

is invariant under the 4-parameter class of transformations

$$
\begin{gathered}
u^{*}=-\frac{a_{1} u}{p+a_{2}}, \quad v^{*}=-\frac{a_{1} v}{p+a_{2}}, \quad p^{*}=a_{4}-\frac{a_{1}^{2} a_{3}}{p+a_{2}}, \\
e^{*}=\frac{a_{3} c^{2}\left(p+a_{2}\right)(e+p) \Delta}{\left(p+a_{2}\right)\left(c^{2}-q^{2}\right)+(e+p) q^{2}}-a_{4}+\frac{a_{1}^{2} a_{3}}{p+a_{2}} \\
\rho^{*}=a_{3} \rho c \Gamma \sqrt{\Delta} \frac{p+a_{2}}{p+S q^{2}+a_{2}},
\end{gathered}
$$

where

$$
\Delta=1-a_{1}^{2} \frac{q^{2}}{c^{2}\left(p+a_{2}\right)^{2}}, \quad S=(e+p) \Gamma^{2},
$$

and

$$
\begin{gathered}
d x^{*}=-\left(p+S v^{2}+a_{2}\right) d x+S u v d y, \quad d y^{*}=S u v d x-\left(p+S u^{2}+a_{2}\right) d y \\
0<\left|J\left(x^{*}, y^{*} ; x, y\right)\right|<\infty
\end{gathered}
$$

In the following, it proves convenient to introduce a scaling $x^{*} \rightarrow a_{1} x^{*}, y^{*} \rightarrow a_{1} y^{*}$ which leaves the preceding theorem unchanged.

Here, we proceed with the specialisation of parameters (4) whence the corresponding subclass of reciprocal transformations becomes

$$
\begin{gathered}
u^{*}=\frac{u}{\epsilon p+1}, \quad v^{*}=\frac{v}{\epsilon p+1}, \quad p^{*}=\frac{p}{\epsilon p+1}, \quad \rho^{*}=\rho c \Gamma \sqrt{\tilde{\Delta}} \frac{\epsilon p+1}{\epsilon\left(p+S q^{2}\right)+1}, \\
e^{*}=\frac{c^{2}(\epsilon p+1)(e+p) \tilde{\Delta}}{(\epsilon p+1)\left(c^{2}-q^{2}\right)+\epsilon(e+p) q^{2}}-\frac{p}{\epsilon p+1}
\end{gathered}
$$


where $\tilde{\Delta}=1-\frac{q^{2}}{c^{2}(\epsilon p+1)^{2}}$, and

$$
d x^{*}=\epsilon\left(\left(p+S v^{2}\right) d x-S u v d y\right) d x+d x, \quad d y^{*}=\epsilon\left(-S u v d x+\left(p+S u^{2}\right) d y\right)+d y,
$$

The preceding relations (16)-(17) constitute a Lie group of transformations in the space of the variables $(\rho, u, v, p, e, d x, d y)$ with the infinitesimal generator

$$
\begin{gathered}
X=-\rho e q^{2} \Gamma^{2} \partial_{\rho}-p u \partial_{u}-p v \partial_{v}-p^{2} \partial_{p}+\left(c^{2} p^{2}-e^{2} q^{2}\right) \Gamma^{2} \partial_{e} \\
+\left(\left(\left(c^{2}-u^{2}\right) p+e v^{2}\right) d x-u v(e+p) d y\right) \Gamma^{2} \partial_{d x}+\left(\left(\left(c^{2}-v^{2}\right) p+e u^{2}\right) d y-u v(e+p) d x\right) \Gamma^{2} \partial_{d y},
\end{gathered}
$$

in that they satisfy the Cauchy problem

$$
\begin{array}{rlrl}
\frac{d \rho^{*}}{d \epsilon} & =-\frac{\rho^{*} e^{*} q^{* 2}}{c^{2}-q^{* 2}}, & \rho_{\mid \epsilon=0}^{*}=\rho, \\
\frac{d u^{*}}{d \epsilon} & =-p^{*} u^{*}, & & u_{\mid \epsilon=0}^{*}=u, \\
\frac{d v^{*}}{d \epsilon_{*}} & =-p^{*} v^{*}, & v_{\mid \epsilon=0}^{*}=v, \\
\frac{d p^{*}}{d \epsilon} & =-p^{* 2}, & & p_{\mid \epsilon=0}^{*}=p, \\
\frac{d e^{*}}{d \epsilon} & =\frac{c^{2} p^{* 2}-q^{* 2} e^{* 2}}{c^{2}-q^{* 2}}, & & e_{\mid \epsilon=0}^{*}=e, \\
\frac{d\left(d x^{*}\right)}{d \epsilon} & =\frac{\left(\left(c^{2}-u^{* 2}\right) p^{*}+e^{*} v^{* 2}\right) d x^{*}-u^{*} v^{*}\left(e^{*}+p^{*}\right) d y^{*}}{c^{2}-q^{* 2}}, & & \left(d x^{*}\right)_{\mid \epsilon=0}=d x, \\
\frac{d\left(d x^{*}\right)}{d \epsilon} & =\frac{\left(\left(c^{2}-v^{* 2}\right) p^{*}+e^{*} u^{* 2}\right) d y^{*}-u^{*} v^{*}\left(e^{*}+p^{*}\right) d x^{*}}{c^{2}-q^{* 2}}, & \left(d x^{*}\right)_{\mid \epsilon=0}=d x .
\end{array}
$$

Particular invariants associated with the one-parameter class of reciprocal transformations (16) are given by

$$
J_{1}=\frac{u}{p}, \quad J_{2}=\frac{v}{p}, \quad J_{3}=\frac{(c p-e q)(c-q)}{(c p+e q)(c+q)}, \quad J_{4}=\rho \frac{J_{3}(c+q)+c-q}{c^{2}-q^{2}} .
$$

\section{Conclusion}

Invariance properties in both nonlinear continuum mechanics and mathematical physics continue to be of research interest. Thus, in particular, invariance under multi-parameter reciprocal transformations in relativistic gasdynamics has recently been established in [36, 37]. Here, a one-parameter subclass of such invariant transformations has been linked to establish Lie group procedures such as set down in the seminal works of [40, 41].

Substitution-type invariance principles as originally derived in spatial gasdynamics by Prim [42] with extension to magnetogasdynamics (see e.g. [43]) may likewise be shown to have a Lie group genesis [44, 45]. Such invariance principles are readily established for the relativistic gasdynamics systems of [36, 37]. In magnetogasdynamics, invariance under multi-parameter reciprocal transformations have been derived in [46] as symmetries 
of an exterior differential system. In separate developments [47, 48] a range of invariant transformations in non-conducting gasdynamics have been derived via matrix formulations. Conjugation of reciprocal-type transformations has recently been applied in [49, 50] to solve nonlinear boundary problems incorporating heterogeneity and relevant to seepage soil mechanics. However, a general investigation via Lie group connections of the conjugation and its application of reciprocal-type invariant transformations to such nonlinear boundary value problems of physical importance remains to be undertaken.

\section{Dedication}

This paper is dedicated to the memory of Nail Ibragimov in recognition of his diverse contributions to the application of Lie group methods.

\section{Acknowledgements}

The research of SVM was supported by the Russian Science Foundation Grant No. 18-1100238 'Hydrodynamics-type equation: symmetries, conservation laws, invariant difference schemes'.

\section{References}

[1] H. Bateman, The lift and drag functions for an elastic fluid in two-dimensional irrotational flow, Proc. Nat. Acad. Sci. 24A (1938) pp.246-251.

[2] H.S. Tsien, Two-dimensional subsonic flow of compressible fluids, J. Aeronautical Sciences 6 (1939) pp.399-407.

[3] S.A. Chaplygin, Collected Works, Gas Jets ANSSR Vol II (1933).

[4] C. Loewner, Generation of solution of partial differential equations by composition of infinitesimal Bäcklund transformations, J. Anal. Math. 2 (1952) pp.219-242.

[5] C. Rogers, W.F. Shadwick, Bäcklund Transformations and Their Applications, Academic Press, Mathematics in Science and Engineering Series, New York (1982).

[6] C. Rogers, W.K. Schief, Bäcklund and Darboux Transformations. Geometry and Modern Applications in Soliton Theory, Cambridge Texts in Applied Mathematics, Cambridge University Press (2002).

[7] C. Rogers, W.K. Schief, K.W. Chow, On a novel class of model constitutive laws in nonlinear elasticity. Construction via Loewner Theory, Theoretical and Mathematical Physics, 152 (2007) pp.711-720.

[8] J.Y. Kazakia, E. Varley, Large amplitude waves in bounded media II. The deformation of an impulsively loaded slab: the first reflexion, Phil. Trans. Roy. Soc. London, A277 (1974) pp.191-237.

[9] T.W. Duerig, A.R. Pelton, D. Stöckel, The use of superelasticity in medicine, Fachzeitschrift für Handel, Wirtschaft, Technik und Wissenshaft, Sonderdruck aus Heft, 9 Metall Verlag-Hüthig (1996) pp.569-574. 
[10] T.W. Duerig, Present and future applications of shape memory and superelastic materials, Mat. Res. Soc. Symp., 360 (1995) pp.497-506.

[11] B.G. Konopelchenko, C. Rogers, On 2+1-dimensional nonlinear systems of Loewnertype, Phys. Lett. A, 158 (1991) pp.391-397.

[12] B.G. Konopelchenko, C. Rogers, On generalised Loewner systems: novel integrable equations in 2+1-dimensions, J. Math. Phys., 34 (1993) pp.214-242.

[13] J.G. Kingston, C. Rogers, Reciprocal Bäcklund transformations of conservation laws, Phys. Lett. A, 92 (1982) pp.261-264.

[14] C. Rogers, P. Wong, On reciprocal Bäcklund transformations of inverse scattering schemes, Physica Scripta, 30 (1984) pp.10-14.

[15] C. Rogers, S. Carillo, On reciprocal properties of the Caudrey-Dodd-Gibbon and Kaup-Kupershmidt hierarchies, Physica Scripta, 36 (1987) pp.865-869.

[16] S. Carillo, B. Fuchssteiner, The abundant symmetry structure of hierarchies of nonlinear equations obtained by reciprocal links, J. Math. Phys., 30 (1989) pp.1606-1613.

[17] A.N.W. Hone, Reciprocal transformations, Painlevé property and solutions of energydependent Schrödinger hierarchies, Phys. Lett. A, 249 (1998) pp.46-54.

[18] C. Rogers, M.C. Nucci, On reciprocal transformations and the Korteweg-de Vries hierarchy, Physica Scripta, 33 (1986) pp.289-292.

[19] B.G. Konopelchenko, C. Rogers, Bäcklund and reciprocal transformations: gauge connections in Nonlinear Equations in Applied Science, Eds. W.F. Ames and C. Rogers, Academic Press (1992) pp.317-362.

[20] K. Tian, Q.P. Liu, Two new supersymmetric equations of Harry Dym type and their supersymmetric reciprocal transformations, Phys. Lett. A, 376 (2012) pp.2334-2340.

[21] W. Oevel, C. Rogers, Gauge transformations and reciprocal links in 2+1-dimensions, Rev. Math. Phys., 5 (1993) pp.299-330.

[22] C. Rogers, Application of a reciprocal transformation to a two-phase Stefan problem, J. Phys. A: Math. Gen., 18 (1985) L105-L109.

[23] C. Rogers, On a class of moving boundary problems in nonlinear heat conduction. Application of a Bäcklund transformation, Int. J. Nonlinear Mechanics, 21 (1986) pp.249-256.

[24] M.L. Storm, Heat conduction in simple metals, J. Appl. Phys., 22 (1951) pp.940-951.

[25] C. Rogers, P. Broadbridge, Sedimentation in a bounded column, Int. J. Nonlinear Mechanics, 27 (1992) pp.661-667.

[26] C. Rogers, P. Broadbridge, On a nonlinear moving boundary problem with heterogeneity: application of a reciprocal transformation, Zeit. angew. Math. Phys., 39 (1988) pp.122-128. 
[27] A.S. Fokas, C. Rogers, W.K. Schief, Evolution of methacrylate distribution during wood saturation. A nonlinear moving boundary problem, Appl. Math. Lett., 18 (2005) pp.321-328.

[28] C. Rogers, Moving boundary problems for the Harry Dym equation and its reciprocal associates, Zeit. angew. Math. Phys., 66 (2015) pp.2069-2079.

[29] L.P. Kadanoff, Exact solutions of the Saffman-Taylor problem with surface tension, Phys. Rev. Lett., 65 (1990) pp.2986-2988.

[30] G.L. Vosconceles, L.P. Kadanoff, Stationary solution for the Saffman-Taylor problem with surface tension, Phys. Rev. A, 44 (1991) pp.6490-6495.

[31] P.J. Vassiliou, Harry Dym Equation in Encyclopaedia of Mathematics (2001) Kluwer Academic Publishers.

[32] N. H. Ibragimov and C. Rogers. On infinitesimal reciprocal-type transformations in gasdynamics. Lie group connections and nonlinear self-adjointness. Ufa Mathematical journal, 4(4):196-207, 2012.

[33] C. Rogers, Y. Huang, On an integrable deformed affinsphären equation. A reciprocal gasdynamic connection, Phys. Lett., A376 (2012) pp.1446-1450.

[34] W.K. Schief, C. Rogers, The affinsphären equation. Moutard and Bäcklund transformations, Inverse Problems, 10 (1994) pp.711-731.

[35] C. Rogers, W.K. Schief, K.W. Chow, C.C. Mak, On Tzitzeica vortex streets and their reciprocals in subsonic gasdynamics, Stud. Appl. Math., 114 (2005) pp.271-283.

[36] C. Rogers and T. Ruggeri. On invariance in 1+1-dimensional isentropic relativistic gasdynamics. Wave Motion, 94:102527, 2020.

[37] C. Rogers, T. Ruggeri, and W. K. Schief. On relativistic gasdynamics: invariance under a class of reciprocal-type transformations and integrable Heisenberg spin connections. Proc. R. Soc. A, 476:20200487, 2020.

[38] C. Rogers, Reciprocal relations in non-steady one-dimensional gasdynamics, Zeit. angew. Math. Phys., 19 (1968) pp.58-63.

[39] S.P. Castell, C. Rogers, Application of invariant transformations in one-dimensional non-steady gasdynamics, Quart. Appl. Math., 32 (1974) pp.241-251.

[40] L. V. Ovsiannikov. Group Analysis of Differential Equations. Nauka, Moscow, 1978. English translation, Ed. W.F. Ames, Academic Press, New York, 1982.

[41] N.K. Ibragimov, Groups of Transformations in Mathematical Physics, Nauka, Moscow (1983).

[42] R.C. Prim, A note on the substitution principle for steady gas flows, J. Applied Phys., 20 (1949) pp.448-450. 
[43] G. Power, C. Rogers, Substitution principles in nonsteady magneto-gasdynamics, Appl. Sci. Res., 21 (1969) pp.176-184.

[44] F. Olivieri, On the equations of ideal gasdynamics with a separable equation of state. Lie group analysis and the Substitution Principle, Int. J. Nonlinear Mech., 27 (1992) pp.773-784.

[45] F. Olivieri, M.P. Speciale, Exact solutions to the ideal magneto-gasdynamics equations through Lie group analysis and substitution principles, J. Phys. A: Math. Gen., 38 (2005) pp.8803-8820.

[46] C. Rogers, J.G. Kingston, W.F. Shadwick, Reciprocal-type transformations in magnetogasdynamics, J. Math. Phys., 21 (1980) pp.395-397.

[47] C. Rogers, Invariant transformations in non-steady gasdynamics and magnetogasdynamics, Zeit. angew. Math. Phys., 20 (1969) pp.370-382.

[48] C. Rogers, The construction of invariant transformations in plane rotational gasdynamics, Arch. Rat. Mech. Anal., 47 (1972) pp.36-46.

[49] C. Rogers, On Stefan-type moving boundary problems with heterogeneity: canonical reduction via conjugation of reciprocal transformations, Acta Mechanica, 230 (2019) pp.839-850.

[50] C. Rogers, P. Broadbridge, On transport through heterogeneous media. Application of conjugated reciprocal transformations, Zeit. angew. Math. Phys., 71 (2020) pp.86. 